\title{
Multifocal osteoarticular tuberculosis of the extremities in an immunocompetent young man without pulmonary disease: A case report
}

\author{
SHUANG HU* , JIA GUO*, TING JI, GUOHUA SHEN and ANREN KUANG \\ Department of Nuclear Medicine, West China Hospital of Sichuan University, Chengdu, Sichuan 610041, P.R. China
}

Received September 3, 2014; Accepted March 19, 2015

DOI: $10.3892 /$ etm.2015.2425

\begin{abstract}
Osteoarticular tuberculosis (TB), an uncommon form of extrapulmonary $\mathrm{TB}$, is a universal mimicker and thus represents a potential differential diagnosis of any osteolytic lesion. The present study describes a case of multifocal osteoarticular TB in an immunocompetent young man who presented with swelling and extremity pain. The patient underwent plain radiography, bone scan and magnetic resonance imaging. A diagnosis was ultimately made based on the pathology results from his second left toe. The patient responded well to anti-TB medication. The radiological findings of multifocal osteoarticular TB are described in this report. To the best of our knowledge, this is the first reported case of multifocal osteoarticular TB symmetrically affecting all the extremities.
\end{abstract}

\section{Introduction}

Osteoarticular tuberculosis (TB), an uncommon form of extrapulmonary TB, comprises $1-6 \%$ of all TB cases and $10-15 \%$ of all extrapulmonary TB cases (1-4). The most frequent sites of osteoarticular TB are the spine, hip and knee. Multifocal skeletal TB is rare and accounts for $10 \%$ of all osteoarticular TB cases (5).

The diagnosis of multifocal osteoarticular TB is often delayed due to the clinical and radiological resemblance of the disease to numerous malignant and bone diseases $(6,7)$. The consequences of considerable diagnostic delays in multifocal osteoarticular TB can prove critical for patients, since such delays may lead to the spread of the infection from the bone to the adjacent joints and surrounding soft tissue, causing

Correspondence to: Dr Anren Kuang, Department of Nuclear Medicine, West China Hospital of Sichuan University, 37 Guo Xue Xiang, Chengdu, Sichuan 610041, P.R. China

E-mail: kuanganren@263.net

*Contributed equally

Key words: tuberculosis, osteoarticular, diagnosis, magnetic resonance imaging, bone scan significant functional disabilities $(8,9)$. The present study describes the case report of a patient that presented with multifocal extremity pain and swellings and, following several TB modalities, was diagnosed with multifocal osteoarticular TB. The patient responded well to anti-TB medication.

\section{Case report}

A 19-year-old Chinese male presented with multifocal painful swellings of his extremities, particularly around the ankles and the interphalangeal and intermetatarsal joints. The patient had been suffering from the painful swelling for $\sim 3$ years and with sinus discharge in certain areas for $\sim 1$ year.

Three years ago, the patient felt pain around his left ankle and several interphalangeal joints of the left foot. Subsequently, some of the lesions began to fester. Similar lesions also appeared symmetrically on the left and right hand and right foot. The patient had a history of pulmonary TB that had been diagnosed 6 years prior to these events; however, the district hospital had confirmed that the condition was cured after 6 months of medication (a 2-month treatment with isoniazid, rifampin and pyrazinamide and a 4-month treatment with isoniazid plus rifampin).

Whilst the patient presented with decreased appetite, he had no history of significant weight loss, night sweats or chronic cough, and he had not taken any immunosuppressive or steroid drugs. The most recent computerized tomography (CT) scans of the patient's chest were normal. In addition, no family members of the patient had presented with the same pattern of symptoms. The patient weighed $50 \mathrm{~kg}$ and appeared to be malnourished.

Upon physical examination, the proximal interphalangeal joint of the patient's thumb, index, middle and little finger of the right hand, thumb of the left hand and right and left feet were swollen. Ulcers were visible on both of the patient's thumbs and on the left foot. The patient was in pain and had difficulty moving his extremities. Laboratory blood-test results showed normal peripheral blood count values and raised erythrocyte sedimentation rate $(75 \mathrm{~mm}$ at the end of $1 \mathrm{~h}$ ). The enzyme-linked immunosorbent assay was negative for human immunodeficiency virus and the radiographs of the thoracolumbar spine, chest and pelvis were normal. The plain radiograph showed soft tissue swelling at the proximal interphalangeal and intermetatarsal joints of the aforementioned 


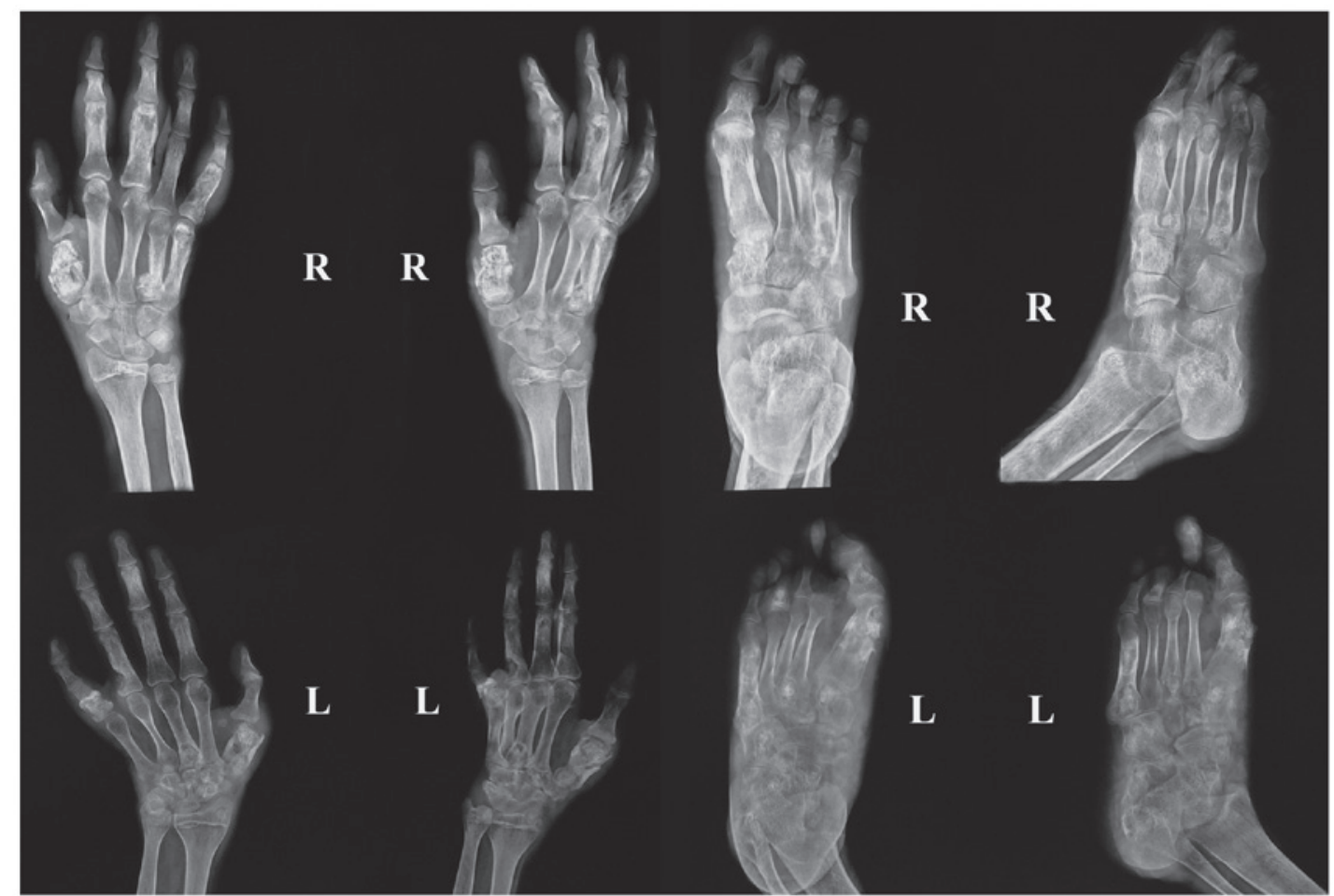

Figure 1. Plain radiographs of the extremities showing lytic and sclerotic lesions of the digits and osteopenia of all bones. L, left; R, right.

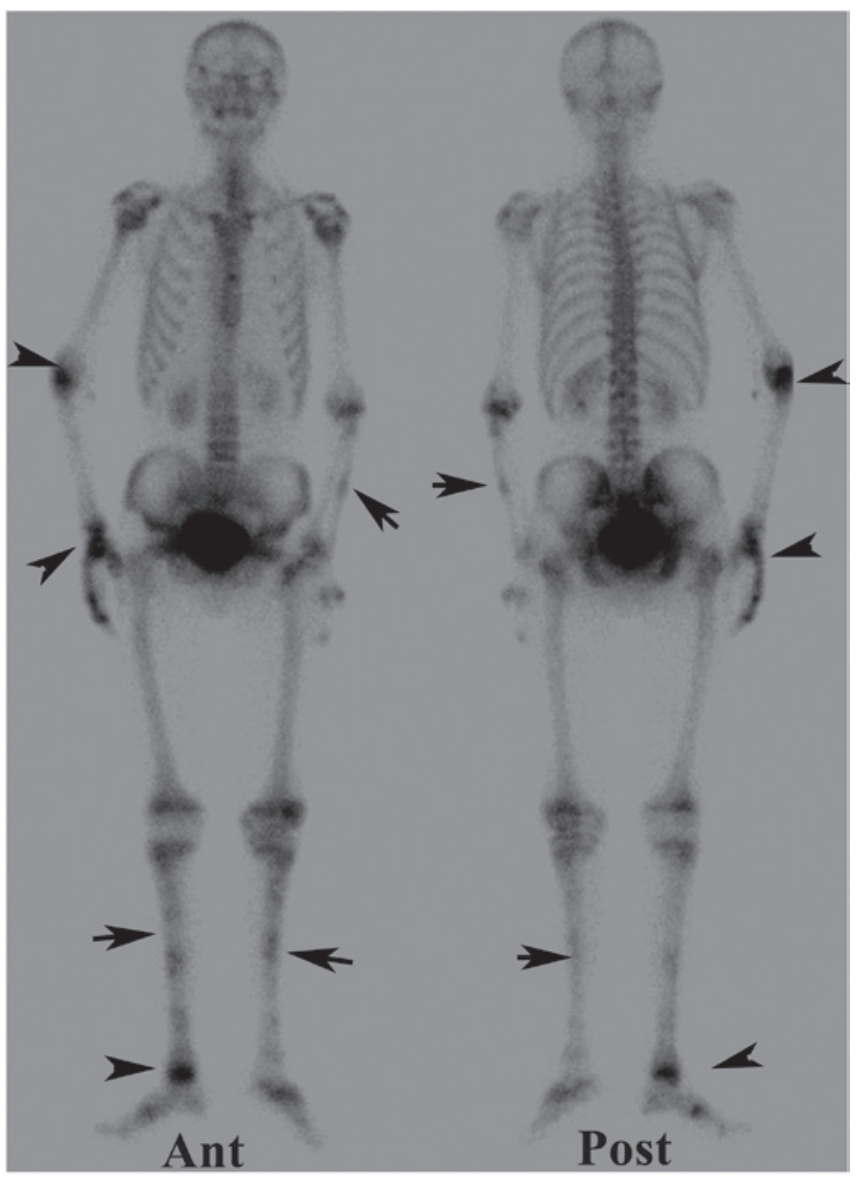

Figure 2. Whole-body bone scan performed $2.5 \mathrm{~h}$ after an intravenous injection of $740 \mathrm{MBq}(20 \mathrm{mCi}) \mathrm{MDP}$ revealed multiple abnormal foci in the right elbow, right wrist and several right interphalangeal and intermetatarsal joints (arrow heads). Low-grade MDP foci can be noted in the tibia, fibula, radius and ulnar on both sides of the body (arrows). MDP, ${ }^{99 \mathrm{~m}} \mathrm{Tc}-\mathrm{methylene}$ diphosphonate; ant, anterior; post, posterior. digits, as well as narrowing of the joints, and several digits presented with sclerosis and osteopenia (Fig. 1). The whole body ${ }^{99 \mathrm{~m}} \mathrm{Tc}-\mathrm{methylene}$ diphosphonate (MDP) bone scan revealed multiple abnormal foci in the extremities (Fig. 2). Magnetic resonance imaging (MRI) of both feet revealed pathological signal intensity changes inside the bones, along with marginal enhancement following contrast injection (Fig. 3).

The biopsy of the second left toe lesion showed a granulomatous lesion with necrosis (including Langhans giant cell and epithelioid cell central caseation), which was suggestive of TB; however the acid-fast stain (Ziehl-Neelsen) showed no acid-fast bacilli (AFB). The diagnosis of TB was confirmed by the polymerase chain reaction (PCR). Anti-TB medication was then prescribed, consisting of isoniazid, rifampin, streptomycin and ethambutol. Compared with the previous prescription of the patient, which consisted of three types of medicine, the new regimen comprised four types, including two new drugs that were added in case of drug resistance. After 3 months of treatment, the lesions gradually healed and the pain and swelling subsided. At present, 2 years after the treatment, the patient can stand on his own feet without any symptoms of pain or swelling.

\section{Discussion}

To the best of our knowledge, this is the first reported case of multifocal osteoarticular TB affecting all the extremities, predominantly the bones. Although no AFB were detected, positive PCR results for Mycobacterium tuberculosis confirmed the diagnosis. Furthermore, the response of the patient to anti-TB medication revealed strong evidence of TB infection. 


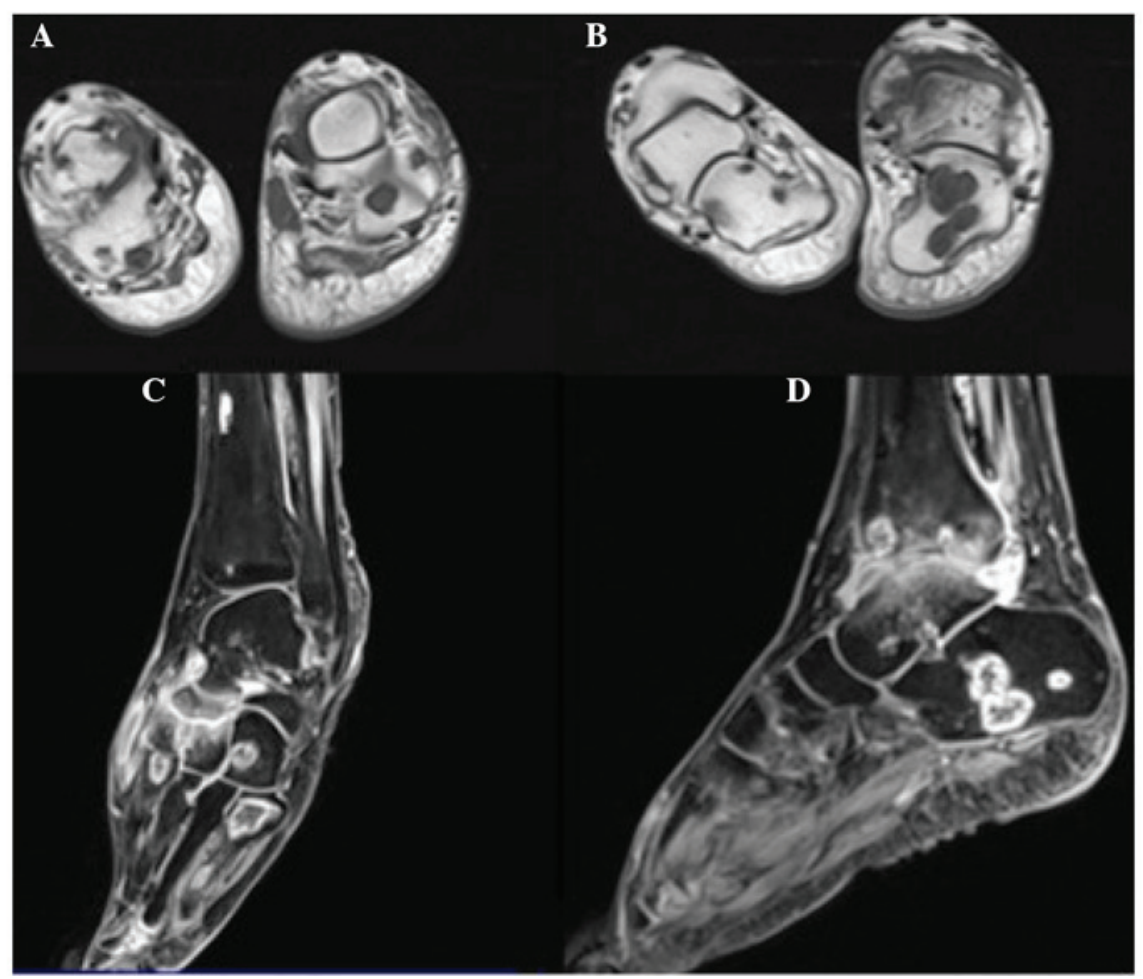

Figure 3. (A and B) Transverse T1-weighted MRI scans of the small bones of the left and right feet showing abnormal hypo-intense signals in or around the bones (black arrows). (C and D) Sagittal contrast-enhanced T1-weighted MRI scans of the (C) left and (D) right feet showing marginal enhancement of the affected lesions (white arrows). The tibias in the scan show pathological signals that correspond to the abnormal foci on the bone scan. MRI, magnetic resonance imaging.

Multifocal skeletal TB most often affects the spine, whereas tuberculous arthritis affects the weight-bearing joints and extraspinal tuberculous osteomyelitis affects the skeletal system, excluding the spine (10). The preference of TB for large joints and the spine can be explained by the rich vascular supply of the vertebra and the growth plates of the long bones, in conjunction with the hematogenous spread of TB (11-14). In the present study, TB was observed in the long and short bones and the small joints of the extremities, while the weight-bearing joints, such as the hips and knees, remained healthy. The exact physiological mechanisms of TB remain unclear and direct inoculation cannot completely explain the symmetry of the afflictions. The presence of lesions in atypical positions cannot exclude a diagnosis of TB.

Osteoarticular TB is a major cause of morbidity. Diagnosis of multifocal skeletal TB is frequently delayed due to its rarity and the considerably vague nature of its symptoms. Furthermore, it is difficult to differentiate multifocal skeletal TB from other bone lesions based on clinical or radiological findings alone (15). In the present study, lytic lesions were observed in the plain radiographs of the extremities of the patient, and multiple MDP foci of varied intensity were identified on the bone scans. Several bone diseases, including Ollier's disease and Maffucci syndrome, were initially considered as potential diagnoses. The high-intensity MDP foci surrounding the right wrist, ankle and interphalangeal and intermetatarsal joints may have been partly due to the fact that the left side of the patient's body was affected first, with the right side consequently becoming weight-bearing and functionally compensating. Another possible explanation is that the infection on the right side was more active.
Signal changes on the MRI scans suggested low-grade bacterial infection. MRI can be used to discriminate between abscesses and granulation tissue, delineate soft-tissue mass and identify the amount of bone destruction (16); however, MRI may not be appropriate for whole-body imaging as it is expensive and time-consuming (17). Bone scans, on the other hand, play an important role in the diagnosis and evaluation of TB $(17,18)$, since they can detect unsuspected osseous TB in patients without known systemic diseases, particularly patients suffering from pain but without known malignancies. Bone scans also help to determine the optimal biopsy site and the most suitable area for insightful MRI imaging. Bone TB should be considered among the differential diagnoses when positive bone scans are observed in patients with unknown causes of bone pain, particularly in patients who do not have a known history of malignancy $(17,19)$.

In conclusion, pain and swelling may be presentations of osteoarticular TB infection. Bone and MRI scans are imaging techniques that have proven to be useful in indicating the extent of the disease, particularly in long bone lesions in the early stage of the disease. Bone scans are also useful in establishing those sites that could require further evaluation by $\mathrm{CT}$, MRI or biopsy.

\section{Acknowledgements}

This study was supported by the National Natural Science Foundation of China (grant no. 81071184) and the Sector Funds of the Ministry of Health of China (grant no. 201002002). 


\section{References}

1. Houston A and Macallan DC: Extrapulmonary tuberculosis. Medicine 42: 18-22, 2014.

2. Jutte PC, van Loenhout-Rooyackers JH, Borgdorff MW and van Horn JR: Increase of bone and joint tuberculosis in The Netherlands. J Bone Joint Surg Br 86: 901-904, 2004.

3. Sandher DS, Al-Jibury M, Paton RW and Ormerod LP: Bone and joint tuberculosis: Cases in Blackburn between 1988 and 2005. J Bone Joint Surg Br 89: 1379-1381, 2007.

4. Hong L, Wu JG, Ding JG, Wang XY, Zheng MH, Fu RQ, et al: Multifocal skeletal tuberculosis: Experience in diagnosis and treatment. Med Mal Infect 40: 6-11, 2010.

5. Tuli SM (ed.): Tuberculous osteomyelitis. In: Tuberculosis of the Skeletal System. 3rd edition. Jaypee Brothers Medical Publishers Ltd., New Delhi, pp174-183, 2004.

6. Go SW, Lee HY, Lim CH, Jee WH, Wang YP, Yoo IeR and Kang JY: Atypical disseminated skeletal tuberculosis mimicking metastasis on PET-CT and MRI. Intern Med 51: 2961-2965, 2012

7. Johnstone RH, Ardern DW and Bartle DR: Multifocal skeletal tuberculosis masquerading as metastatic disease. ANZ J Surg 81: 731-733, 2011.

8. Cheung PY, Ho KW, Lam YL and Shek TW: Unusual presentations of osteoarticular tuberculosis in two paediatric patients BMJ Case Rep 2012: bcr2012006714, 2012.

9. Dhillon MS and Tuli SM: Osteoarticular tuberculosis of the foot and ankle. Foot Ankle In 22: 679-686, 2001.
10. Moore SL and Rafii M: Imaging of musculoskeletal and spinal tuberculosis. Radiol Clin North Am 39: 329-342, 2001.

11. Gardam M and Lim S: Mycobacterial osteomyelitis and arthritis. Infect Dis Clin North Am 19: 819-830, 2005.

12. Tuli SM: General principles of osteoarticular tuberculosis. Clin Orthop Relat Res 39: 11-19, 2002.

13. Magnussen A, Dinneen A and Ramesh P: Osteoarticular tuberculosis: Increasing incidence of a difficult clinical diagnosis. Br J Gen Pract 63: 385-386, 2013.

14. Scanzello CR and Goldring SR: The role of synovitis in osteoarthritis pathogenesis. Bone 51: 249-257, 2012.

15. Marudanayagam A and Gnanadoss JJ: Multifocal skeletal tuberculosis: A report of three cases. Iowa Orthop J 26: 151-153, 2006.

16. Teo HE and Peh WC: Skeletal tuberculosis in children. Pediatr Radiol 34: 853-860, 2004.

17. Zhang Y, Zhang Y and Ma J: The prospect of incidental detection of unsuspected skeletal tuberculosis by bone scintigraphy should not be overlooked. Clin Nucl Med 32: 435-439, 2007.

18. Trikha V, Gupta V, Rastogi S and Kumar R: Tuberculosis of calcaneus: Assessing treatment response by Tc-99m MDP scintigraphy. Clin Nucl Med 29: 506, 2004.

19. Bhardwaj V, Agrawal M, Suri T, Sural S, Kashyap R and Dhal A: Evaluation of adequacy of short-course chemotherapy for extraspinal osteoarticular tuberculosis using $99 \mathrm{mTc}$ ciprofloxacin scan. Int Orthop 35: 1869-1874, 2011. 